\section{Is fracture risk increased in patients with coeliac disease?}

\section{J Compston}

\section{Concerns about fracture risk in patients with coeliac disease may have been exaggerated}

M etabolic bone disease is a well established complication of coeliac disease. Early reports focused mainly on the association with vitamin D deficiency and osteomalacia but more recently attention has turned to osteoporosis. ${ }^{2}$ A number of studies have demonstrated reduced bone mineral density in individuals with coeliac disease, particularly in untreated cases and in those who fail to respond fully to a gluten free diet. ${ }^{3}$ Furthermore, the need to consider coeliac disease as a pathogenetic factor in individuals presenting with osteoporosis has been emphasised; this particularly applies to those with clinical features of the disease and those who fail to respond to treatment for their osteoporosis.

The clinical significance of the observed reduction in bone mineral density associated with coeliac disease is unclear. While a small proportion of patients undoubtedly develop severe osteoporosis with multiple fragility fractures, the question of whether fracture risk is significantly increased across the clinical spectrum of coeliac disease has only recently been explored. Studies in small numbers of selected patients, drawn from hospital clinics, have suggested that fracture risk is increased ${ }^{4}$; however such studies are likely to overestimate risk and cannot be generalised to the coeliac population as a whole.

A more representative approach is to study fracture rates in population based cohorts of coeliac disease patients and controls. In this issue of Gut, Thomason and colleagues ${ }^{6}$ report the results of one such study in 274 patients and 224 control subjects [see page 518]; no significance difference in fracture risk was demonstrated between these two groups, the overall age and sex adjusted odds ratio being 1.05 (95\% CI 0.66-2.25). For forearm or wrist fractures and for low trauma fractures in men, there was a trend towards increased risk with odds ratios of 1.21 and 1.28 , respectively, but these were not statistically significant. In a larger population based case control study of 1021 patients with coeliac disease, Vestergaard and Mosekilde ${ }^{7}$ also found no significant increase in fracture risk either before or after diagnosis, with incidence rate ratios of 1.15 (1.00-1.32) and 1.19 (1.06-1.33), respectively.

These two studies thus provide no definitive evidence for increased fracture rate in association with coeliac disease. However, the slight (but non-significant) increase in risk observed in both studies might suggest that the failure to demonstrate statistical significance was due to limitations of the study design rather than to the true absence of an association. Important considerations in this respect are the size of the study and the robustness of the methods used for ascertainment of coeliac disease and fracture. Power calculations should take into account all of these factors as poor ascertainment of disease status and/or fracture affects the sample size required. Furthermore, the validity of the power calculations depends critically on the assumed fracture rate in the control population, which in reality varies considerably between studies. The study of Thomason and colleagues ${ }^{6}$ was powered to demonstrate a twofold or greater increase in fracture risk in the coeliac population but did not have sufficient power to demonstrate smaller increases in risk. In contrast, the study of Vestergaard and Mosekilde ${ }^{7}$ was powered to show an increase in incidence risk ratio of 1.2-1.5; however, the relatively low validity $(78 \%)$ of the diagnostic criteria used for coeliac disease is likely to have reduced the power of the study and may have led to underestimation of risk ratios.

While an increase in fracture risk cannot be excluded on the basis of these studies, it can be concluded that the magnitude of any increase, if present, is small and thus for the majority of individuals with coeliac disease absolute risk of fracture is low. Furthermore, prospective studies have shown significant improvement in bone mineral density after introduction of a gluten free diet, ${ }^{38}$ indicating that bone densitometry at diagnosis may considerably overestimate short term fracture probability. This has implications for clinical practice as bone densitometry for all patients with coeliac disease, as advocated in some guidelines, ${ }^{9}$ will not be cost effective and may result in unnecessary treatment with bone preserving agents. A more rational approach is to restrict bone densitometry to the minority of individuals in whom short term (5-10 years) fracture probability is high; risk factors for fracture have not been specifically identified in coeliac disease but are likely to include non- compliance with or failure to respond fully to a gluten free diet, glucocorticoid therapy, untreated hypogonadism, age, low body mass index, and previous fragility fracture.

Effective treatment strategies for osteoporosis in individuals with coeliac disease have not been defined. However, it seems reasonable to advocate similar lifestyle measures to those recommended in postmenopausal osteoporosis $^{10}$; these include vitamin D repletion where required and maintenance of adequate calcium intake, using calcium supplements if necessary. In osteoporotic patients with malabsorption, intravenous therapy with pamidronate or zoledronate should be considered; in others, the therapeutic options should be based on evidence from studies in postmenopausal women and men with osteoporosis.

At the present time therefore available evidence indicates that the risk of fragility fractures is, at most, only slightly increased in coeliac disease and that the absolute risk of fracture in the majority of these individuals is low. Bone densitometry should therefore be reserved for the minority with a high fracture probability, selected on the basis of risk factors. Screening of all patients with coeliac disease, as currently recommended in the British Society of Gastroenterology guidelines, cannot be justified and represents an inappropriate use of resources. The existing guidelines should therefore be revised to accommodate these considerations, which also apply to patients with inflammatory bowel disease. $^{11}$

\section{Gut 2003;52:459-460}

\section{Author's affiliation}

J Compston, Department of Medicine, University of Cambridge School of Clinical Medicine, Cambridge, UK

Correspondence to: Box 157, Department of Medicine, Addenbrooke's Hospital, Cambridge CB2 2QQ, UK; jec1001@cam.ac.uk

\section{REFERENCES}

1 Bennett TI, Hunter D, Vaughan JM. Idiopathic steatorrhoea (Gee's disease). A nutritional disturbance associated with tetany, osteomalacia, and anaemia. Q J Med 1932;4:603-47.

2 Pistorius LR, Sweidan WH, Purdie DW, et al. Coeliac disease and bone mineral density in adult female patients. Gut 1995;37:639-42. 3 McFarlane XA, Bhalla AK, Reeves DR, et al. 3 McFarlane XA, Bhalla AK, Reeves DR, et al.
Osteoporosis in treated coeliac disease. Gut 1995:36:710-14. 


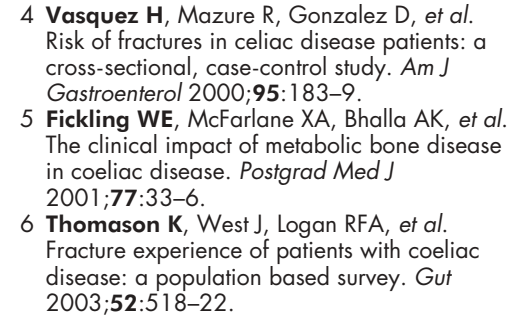

7 Vestergaard P, Mosekilde L. Fracture risk in patients with celiac disease, Crohn's disease, pand ulcerativ with colitis: a nationwide follow-up study of 16,416 patients in Denmark. Am J Epidemiol 2002;156:1-10.

8 Kemppainen T, Kroger H, Janatuinen, E et al. Bone recovery after a gluten-free diet: a 5 -year follow-up study. Bone 1999;25:355-60.

9 Scott EM, Gaywood I, Scott BB. Guidelines for osteoporosis in coeliac disease and inflammatory bowel disease. Gut 2000;46(suppl 1):1-8.
10 Royal College of Physicians and Bone and Tooth Society of Great Britain.

Osteoporosis. Clinical Guidelines for Prevention and Treatment. Update on Pharmacological Interventions and an Algorithm for Management. London: Royal College of Physicians, 2000.

11 Compston JE. Can biochemical markers be used to screen patients with inflammatory bowel disease for osteoporosis? Eur J Gastroenterol Hepatol 2002;14:587-9.

\section{Crohn's disease or Crohn's diseases?}

\section{D R Arnott, J Satsangi}

\section{Disease behaviour in Crohn's disease is dynamic and not stable over time}

n their landmark paper of 1932, Crohn, Ginzburg, and Oppenheimer ${ }^{1}$ described "a disease of the terminal ileum, affecting mainly young adults and characterised by a subacute or chronic necrotising and cicratrising inflammation. The ulceration of the mucosa is accompanied by a disproportionate connective tissue reaction ... which frequently leads to stenosis of the lumen of the intestine, associated with the formation of multiple fistulas". In the intervening years however, the term Crohn's disease has been introduced, and now covers a heterogeneous range of clinical presentations, including the classical phenotype of regional ileitis. A number of attempts have been made to subclassify patients with Crohn's disease into subgroups with similar stable phenotypic characteristics. These attempts have been catalysed first by attempts to individualise therapy, and most recently by progress in understanding the molecular genetics of Crohn's disease, and the need to relate genotype to disease phenotype. Indeed, it is increasingly clear that assigning disease phenotype may be difficult, but critical in determining the successful outcome of genetic association and linkage studies. The experience of the Toronto group is particularly instructive in this respect, demonstrating that the erroneous assignation of phenotype may lead to a $40 \%$ loss of power in linkage studies. ${ }^{2}$

The Vienna classification of the working party of the World Congress of Gastroenterology proposed a subclassification of Crohn's disease according to three overriding phenotypic characteristics, implicated in detailed studies of populations in Europe and North America-age, location of disease, and disease behaviour. ${ }^{3}$ It is the subclassification of disease behaviour into inflammatory (non-stricturing non-penetrating), stricturing (stenosing), and fistulating (penetrating) disease that has been analysed carefully in recent months. Three publications have examined the stability of disease behaviour over time. In the largest study, Cosnes et al analysed data concerning the long term evolution of disease behaviour in 2002 patients with Crohn's disease retrospectively, and performed a five year prospective analysis of a subgroup of 646 patients. ${ }^{4}$ These investigators reported that after 20 years, as many as $88 \%$ of the initial cohort had developed either stricturing $(18 \%)$ or penetrating $(70 \%)$ disease behaviour. In this study, disease location was the most important factor identified in determining an alteration in disease behaviour, with small bowel and anoperineal involvement predicting early stricturing and/or penetrating complications.

Louis et al examined the stability of phenotype in a Belgian population of patients with Crohn's disease followed for up to 25 years. $^{5}$ Again, striking changes in disease behaviour over time were noted. Within 10 years, $46 \%$ of patients had changed disease behaviour from purely inflammatory disease to either stricturing $(27.1 \%$; $<0.0001)$ or penetrating $(29.4 \% ; \mathrm{p}<0.0001)$ disease. Relative to this finding, disease location was stable, although this also changed in approximately $15 \%$ of patients by 10 years.

In the present issue of Gut, Louis and colleagues ${ }^{6}$ have now examined in detail the factors which may influence the progression of disease behaviour [ see page 552]. The authors examined 163 patients with an initial inflammatory (B1) phenotype and assessed changes in behaviour over a five year period. These changes were related to clinical characteristics, NOD2/CARD15 genotype, and serological markers by univariate and multivariate analyses. The authors suggest, consistent with previous studies, that disease location is a critical determinant of progression of disease behaviour. Smoking was a determinant in patients progressing to penetrating complications. Although familial disease distinguished stricturing and penetrating disease, the NOD2/CARD15 genotype was not thought to be a determinant of disease behaviour by the authors.

The major message that emerges from these studies is that disease behaviour is dynamic and not stable over time. Although inflammatory disease is the commonest phenotype at diagnosis, there appears to be an inexorable progression to either stricturing or penetrating disease over time. Approximately $25 \%$ of patients with inflammatory disease type at diagnosis will progress to penetrating disease over a five year period, again consistent with previous data from Belgium and France.

Although the progression may be inevitable, analysis of factors that influence the progression to either stricturing or penetrating disease is clearly of great interest. Cigarette smoking is arguably the most important environmental factor identified in inflammatory bowel disease pathogenesis thus far, with consistent literature emphasising the discordant effect on ulcerative colitis and Crohn's disease. In Crohn's disease, cigarette smoking not only increases disease susceptibility, but also has a well documented detrimental effect on disease course, success of medical therapy, need for repeat surgery, and overall mortality. Recent data even implicate smoking in Infliximab resistance. ${ }^{8}$ We should therefore not be surprised when it is also implicated in the progression from inflammatory to penetrating disease, a phenotype generally considered more severe. ${ }^{9}$ In the present study it is remarkable that the effect is seen even in relatively light smokers (one cigarette per day was the definition used). There are clearly many unanswered questions, of which perhaps the most pertinent are 
the mechanisms involved and the constituent of cigarette smoke that is pathogenic. It is at present unclear why the effect is on the progression to penetrating rather than stricturing disease.

Anatomical location of disease is also strongly implicated in the present study, mirroring previous data. Patients with ileal disease tended to have stricturing disease whereas those with colonic and perianal disease had penetrating complications.

What then of genetic influences on disease behaviour? Germline variants of the NOD2/CARD15 gene are reported in up to $50 \%$ of European patients, ${ }^{10}$ and since the initial identification of this gene, many investigators have performed genotype-phenotype analyses. Different populations and ethnic groups have been studied, and various phenotypic classifications have been used in analysis. The current study failed to identify an association between NOD2/CARD15 status and disease behaviour. In fact, these findings contrast with the three largest published studies which have demonstrated that carriage of one or more NOD2 allelic variants may protect against penetrating disease, and predispose towards stricturing disease. ${ }^{10-12}$ These cited studies and others have also demonstrated an association with ileal involvement; indeed Ahmad and colleagues $^{11}$ suggest strongly that the primary association is with location of disease rather than behaviour per se. The Vienna classification was not used to classify disease behaviour in these previous studies. Nevertheless, many aspects of the definitions are broadly similar, and many conclusions consistent. Why do the present data appear inconsistent with these other studies? There are clearly a number of possible explanations, including ethnic variation and phenotypic definitions. Further inspection of the authors' own data is also revealing and may be most pertinent. Only $62 \%$ of the study population were genotyped, and the numbers in each group (B1, B2, B3) were relatively small. The authors chose to compare carriage rates in inflammatory (B1) versus stricturing (B2) versus fistulising (B3) disease at five years. Further analysis of the same data, comparing patients with fistulising disease (B3) against those who did not develop fistulising complications $(\mathrm{B} 1+\mathrm{B} 2)$ showed a modest protective effect of NOD2/CARD15 carriage $\left(\chi^{2}=4.3, \mathrm{p}<0.04\right)$. NOD2 therefore may be an influential factor in the progression of disease type over time, but may or may not reflect disease phenotype at one time point in diagnosis. To our mind it remains unclear whether the primary association of NOD2 genotype is with disease location or behaviour, or whether these are indeed independent.

The influence of current therapiesimmunosuppressants, and especially the novel biological treatments now commonly used in Crohn's disease-remains largely unknown. A relatively small percentage of patients in the present study received azathioprine, and there is no evidence that any received Infliximab.

There is a great need for accurate subclassification of Crohn's disease, notwithstanding the great difficulties involved. It is clear from the present study and others cited that disease behaviour is dynamic, and needs to be viewed as a continuing progress rather than as a snapshot. There are clearly a number of influences on disease progression, and the present study provides a positive step towards identification of disease factors. The consistency of phenotypic data available may be the greatest challenge to progress in unravelling disease complexities in these studies.

Gut 2003;52:460-461
.

Authors' affiliations

I D R Arnott, J Satsangi, Gastrointestinal Unit, University Department of Medical Sciences, Western General Hospital, Edinburgh, UK

Correspondence to: Professor J Satsangi, Gastrointestinal Unit, Western General Hospital, Crewe Road, Edinburgh EH4 2XU, UK;

J.Satsangi@ed.ac.uk

\section{REFERENCES}

1 Crohn BB, Ginsburg L, Oppenheimer GD. Regional ileitis: A pathological and clinical entity. JAMA 1932;99:1323-9.

2 Silverberg MS, Daly M, Moskovitz DN, et al. Diagnostic misclassification reduces the ability to detect linkage in inflammatory bowe disease genetic studies. Gut 2001:49:773-6.

3 Gasche C, Scholmerich J, Brynskov J, et al. A simple classification of Crohn's disease: report simple classification of Crohn's diseas
of the Working Party for the World Congresses of Gastroenterology, Vienna 1998. Inflamm Bowel Dis 2000;6:8-15.

4 Cosnes J, Cattan S, Blain A, et al. Long-term evolution of disease behavior of Crohn's disease. Inflamm Bowel Dis 2002;8:244-50.

5 Louis E, Collard A, Oger AF, et al. Behaviour of Crohn's disease according to the Vienna classification: changing pattern over the course of the disease. Gut 2001;49:777-82.

6 Louis E, Michel V, Hugot JP, et al. Early development of stricturing or penetrating pattern in Crohn's disease is influenced by disease location, number of flares, and smoking but not by NOD2/CARD 15 genotype. Gut 2003:52:552-7.

7 Rubin DT, Hanauer SB. Smoking and inflammatory bowel disease. Eur J Gastroenterol Hepatol 2000; 12:855-62.

8 Parsi MA, Achkar JP, Richardson S, et al. Predictors of response to infliximab in patients with Crohn's disease. Gastroenterology 2002; 123:707-13.

9 Lautenbach E, Berlin JA, Lichtenstein GR. Risk factors for early postoperative recurrence of Crohn's disease. Gastroenterology 1998; 115:259-67.

10 Lesage S, Zouali H, Cezard JP, et al CARD 15/NOD2 mutational analysis and genotype-phenotype correlation in 612 patients with inflammatory bowel disease. Am J Hum Genet 2002:70:845-57.

11 Ahmad T, Armuzzi A, Bunce M, et al. The Ahmad T, Armuzzi $A$, Bunce $M$, et al.
molecular classification of the clinical manifestations of Crohn's disease. Gastroenterology 2002; 122:854-66.

12 Abreu MT, Taylor KD, Lin YC, et al. Mutations in NOD2 are associated with fibrostenosing disease in patients with Crohn's disease. Gastroenterology 2002;123:679_ 88. 